\title{
Malaria in Meghalaya: a systematic literature review and analysis of data from the National Vector-Borne Disease Control Programme
}

\author{
Anne Kessler ${ }^{1}$, Anna Maria van Eijk ${ }^{1}$, Limalemla Jamir ${ }^{2}$, Catherine Walton $^{3}$, Jane M. Carlton ${ }^{*}$ \\ and Sandra Albert ${ }^{2,4}$
}

\begin{abstract}
Background: Meghalaya, one of eight states in the northeastern region of India, has been reported to carry a high malaria burden. However, malaria surveillance, epidemiology, and vector studies are sparse, and no reviews combining these topics with malaria prevention and control strategies have been published in recent years. Furthermore, no analysis of surveillance data has been published documenting the changes in epidemiology following the first distribution of long-lasting insecticidal nets (LLINs) statewide in 2016.

Methods: A hybrid approach was used to describe the status of malaria in Meghalaya. First, a literature search was performed using the terms 'malaria' and 'Meghalaya'. Second, data were obtained from the Meghalaya State Malaria Control Programme for 2006-2017 for analysis of trends. Data from 3 years 2015-2017 were analysed further by district and year to assess changes in malaria incidence and distribution following the introduction of LLINs.
\end{abstract}

Results/conclusions: Like malaria in mainland India, malaria in Meghalaya is complex, with both Plasmodium falciparum and Plasmodium vivax parasites in circulation, multiple Anopheles vector species, and reports of both unusual and severe malaria syndromes across all age groups. Integrated statewide malaria epidemiology, vector, and prevention and control data for Meghalaya are not readily available, and published studies are largely focused on a single topic or a single district or region of the state. Although malaria prevention and control approaches are available, (e.g. spraying, LLINs, personal repellents), their use and effectiveness is also not well characterized in the literature. Analysis of state malaria control programme data indicates that case incidence and related fatalities in Meghalaya have declined over the last decade. This could be attributed to changes in treatment guidelines and/or statewide distribution of effective prevention methods such as LLINs. Since the distribution of more than 900,000 LLINs in 2016, the malaria caseload has declined significantly in most Meghalaya districts, excluding the remote and geographically isolated South Garo Hills. Additionally, the proportion of adult malaria cases (15+ years of age versus children 0-14 years) in most districts was significantly greater following LLIN distribution, which likely reflects common lifestyle practices in these areas (e.g. adults working during night hours; small children in the households receiving priority for bed net protection). While reduction in malaria case incidence and related deaths is clear, the changes in malaria transmission and clinical manifestation have not been characterized. Routine epidemiology and vector surveillance combined with real-time data reporting are essential for the continued reduction and eventual elimination of malaria in Meghalaya.

Keywords: Malaria, Meghalaya, LLINs, Epidemiology, Anopheles, Complex malaria

\footnotetext{
*Correspondence: jane.carlton@nyu.edu

${ }^{1}$ Center for Genomics and Systems Biology, Department of Biology, New

York University, New York, NY, USA

Full list of author information is available at the end of the article
} 


\section{Background}

Malaria has long been problematic in the mountainous state of Meghalaya [1], one of the eight northeastern states of India, known for their hilly, forested, and inaccessible regions, and for the indigenous/tribal people that live there. Although 'indigenous peoples' is a global term widely used in the literature, it is a contested term in India [2], and the Indian government instead uses the term 'tribals', or the constitutionally-recognized category of 'Scheduled Tribes' to refer to these communities in a countrywide sense [3]. Tribal communities in India are particularly prone to malaria because of their geographical marginalization, poor access to health care, low socioeconomic status, and social factors including cultural and religious values, and beliefs [1, 4]. In Meghalaya, 86\% of the state's $\sim 3.5$ million people belong to scheduled tribes [5]. Most belong to the Khasi-Jaintia and/or Garo tribes, both of which are matrilineal, e.g., children take their mother's name, and the youngest daughter inherits the ancestral land and looks after her parents in their elderly years. The state is divided into eleven districts: Garo Hills (North, East, West, South, South West), Khasi Hills (East, South West, West), Jaintia Hills (East, West), and Ri Bhoi, and each district houses a district hospital, community health centre/s $(\mathrm{CHC})$, and primary health centres (PHCs) and sub-centres that service the inhabitants. Migration within and between the northeastern states is common and likely affects the malaria situation in Meghalaya.

Meghalaya is situated within the Indo-Burma biodiversity hotspot which spans from eastern Bangladesh through northeast India to Myanmar, southern China, Thailand, Lao PDR, Cambodia, Vietnam, the Andaman Islands, and Hainan [6]. The northeast comprise one of the distinct biogeographic regions of India with many endemic flora and fauna [7]. The very high rainfall of northeast India provides ideal conditions for growth of tropical forests that have historically covered the region. However, there has been substantial forest loss and fragmentation over recent decades in Meghalaya and the northeastern states of Tripura and Nagaland, primarily due to widespread shifting cultivation ('jhum') [8]. Although Meghalaya had 69\% forest cover in 1999, a shift from closed to more open forest structure has occurred, particularly in the Khasi and Jaintia Hills [8]. Throughout much of Meghalaya, the forest cover forms a mosaic with a rice-agroecosystem such that villages in a malaria endemic area may be situated close to forests or rice fields or both. The diverse habitats this provides for multiple Anopheles species plays a large role in shaping the current and changing malaria epidemiology of this region.
Although malaria in India is a long-standing public health concern and a central focus of the National Vector Borne Disease Control Programme (NVBDCP), the government agency for the prevention and control of vector-borne diseases, there is a paucity of published data regarding malaria epidemiology, vectors, and control methods, and indeed health systems research in general [9], specific to Meghalaya. Over the past decade, malaria caused by the dominant species Plasmodium falciparum and Plasmodium vivax has been high and unstable across the state. From 2012, there was a steady increase in the number of malaria cases and deaths; however, cases have been decreasing since 2015. Meghalaya malaria control programme data suggests that the distribution of more than 900,000 long-lasting insecticide-treated bed nets (LLINs), distributed for the first time in 2016, could be responsible for the observed decline.

While malaria surveillance and reporting has been largely neglected across all eight Northeastern Indian states, the diversity and complexity of malaria as well as land cover, ecology, and host genetics of indigenous tribes specific to Meghalaya warrants independent assessment. Thus, the objectives of this review were twofold: first, to undertake a literature review of malaria in Meghalaya, with the goal of summarizing what is known about malaria epidemiology, vectors, and prevention methods in the state; and second, to undertake an analysis of statewide malaria epidemiology data from 2015 to 2017 to evaluate the effect of the introduction of LLINs. Malaria in Meghalaya is complex, with both P. falciparum and $P$. vivax prevalent, a plethora of key and emerging Anopheles vectors, severe and unusual clinical syndromes across age groups, and varied use and acceptance of different malaria prevention methods. Published studies focused on single districts have made generalization of malaria epidemiology across the state problematic; however in combination with analysis of Meghalaya State Malaria Control Programme surveillance they provide a solid foundation for continued malaria prevention, control, and elimination efforts.

\section{Methods}

\section{Literature search strategy and selection criteria}

To evaluate existing literature, PubMed and Google Scholar searches were performed using the search terms 'Meghalaya' and 'malaria'. Studies were triaged for inclusion, and those which mentioned Meghalaya as a neighbouring state or case reports not relevant to malaria in Meghalaya were excluded. Case studies were excluded if the central focus was not a malaria diagnosis in Meghalaya. No limit on period of study was enforced when selecting papers for inclusion. 
Extraction and analysis of 'Malaria in Meghalaya' literature Articles meeting the selection criteria were divided into three main categories: malaria epidemiology, malaria vectors, and malaria prevention and control. Within each category, sub-topics were identified and summarized as follows: malaria epidemiology: case incidence, distributions, disease presentations, fatality rates, and reporting; malaria vectors: key species, emerging species, vector diversity, and transmission factors; malaria prevention and control methods: use, effectiveness, and additional factors to consider for improved prevention. All papers selected for inclusion were read, summarized, classified by category, and discussed by at least two authors (AK and AVE).

\section{NVBDCP malaria surveillance data and analysis}

District-wise epidemiology data from 2006 to 2017 were obtained from the Meghalaya NVBDCP and the primary health centres and sub-centres under their jurisdiction. Specifically, data detailing total malaria cases (both $P$. falciparum and $P$. vivax), malaria attributed deaths, and API was obtained for all districts. Age and sex distributions were available for malaria case and fatality data, while species-specific data were only available for the former. Timewise data from the last 3 years, 2015-2017, were obtained to assess and summarize the impact of LLINs distributed statewide for the first time in 2016. The statewide malaria burden and distribution in 2016 is provided for reference in Fig. 1. Information from East and West Jaintia Hills was combined for the analysis, and the data collected for East Khasi Hills were excluded because of the absence of malaria in most of the district, a result of the district's high elevation. For all other districts, data from all PHCs and subcentres was obtained and included in the analysis. The dataset was analysed in Stata 14.2, and summary figures were generated using Microsoft Excel. Statistical analysis was performed using Stata 14.2 or Prism 7.0. Statistical differences in clinical malaria by (1) age $(15+$ years versus children 0-14 years) and (2) sex (proportion of males) between the years preceding (e.g. 2015) and following (e.g. 2017) LLIN distribution were determined

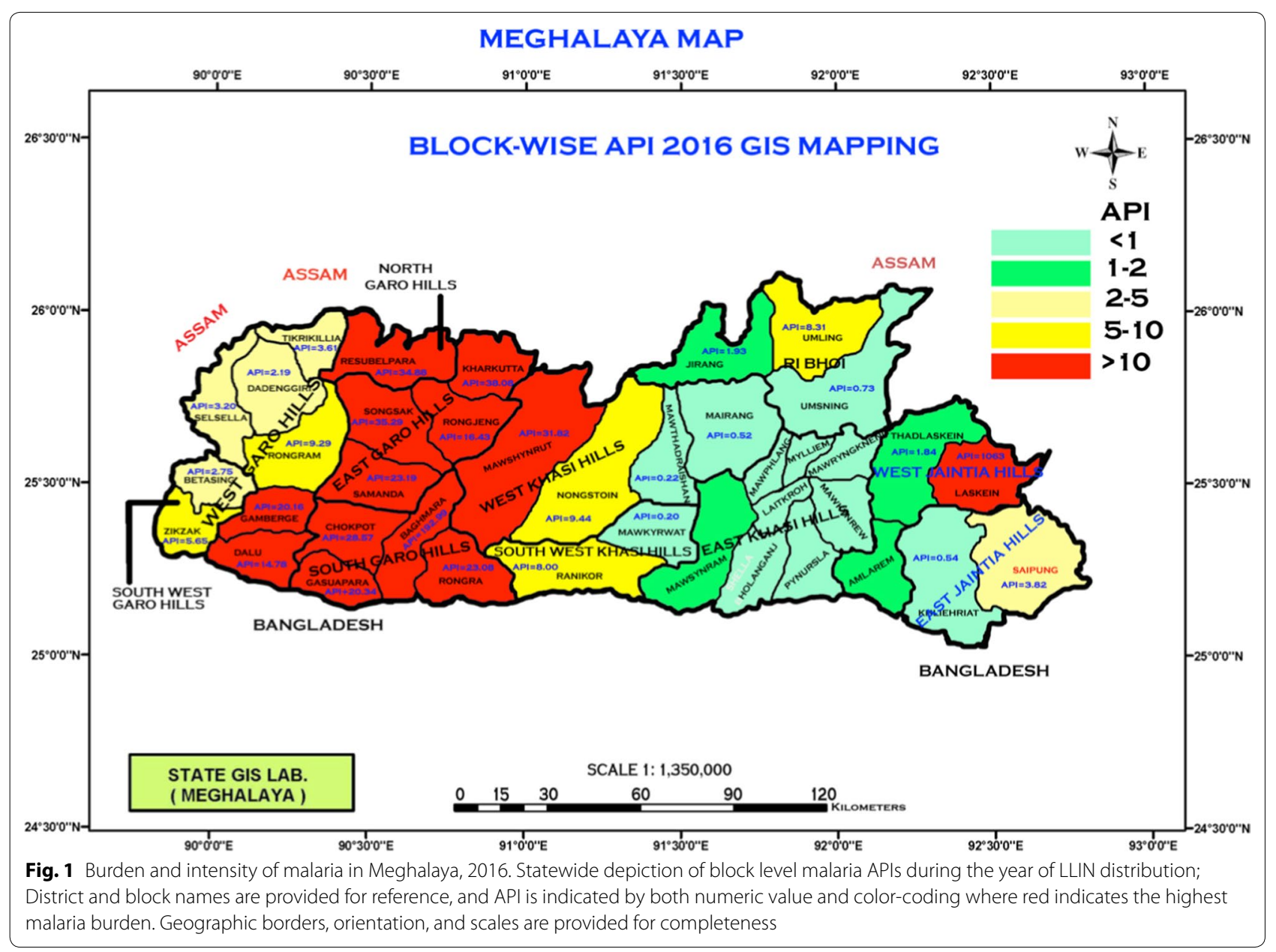


by Chi square tests. Statistical significance was assessed using an $\alpha$ level $<0.05$.

\section{Results}

The literature searches in PubMed and Google Scholar yielded 37 articles (Fig. 2). Of these, 12 were immediately excluded for not meeting inclusion criteria. Specifically, four articles contained no relevant information (e.g., the topic of the article was not malaria in Meghalaya), three were case reports where malaria was not the central focus or diagnosis, and five were excluded for mentioning Meghalaya as a neighboring state, country, or another state on the Indian subcontinent. Additionally, the full text of seven articles could not be accessed (either in the USA or in India), and these articles were also excluded. In total, 18 articles focused on malaria epidemiology, vectors, and/or malaria prevention and control measures were included in the final review (Fig. 2).

\section{Literature review of malaria epidemiology in Meghalaya}

Published reports of malaria epidemiology in Meghalaya described malaria transmission as perennial and persistent across the state $[10,11]$ and at border regions [12]. A retrospective analysis of malaria cases from 2001 to 2009 showed seasonal spikes in malaria, concurrent with the

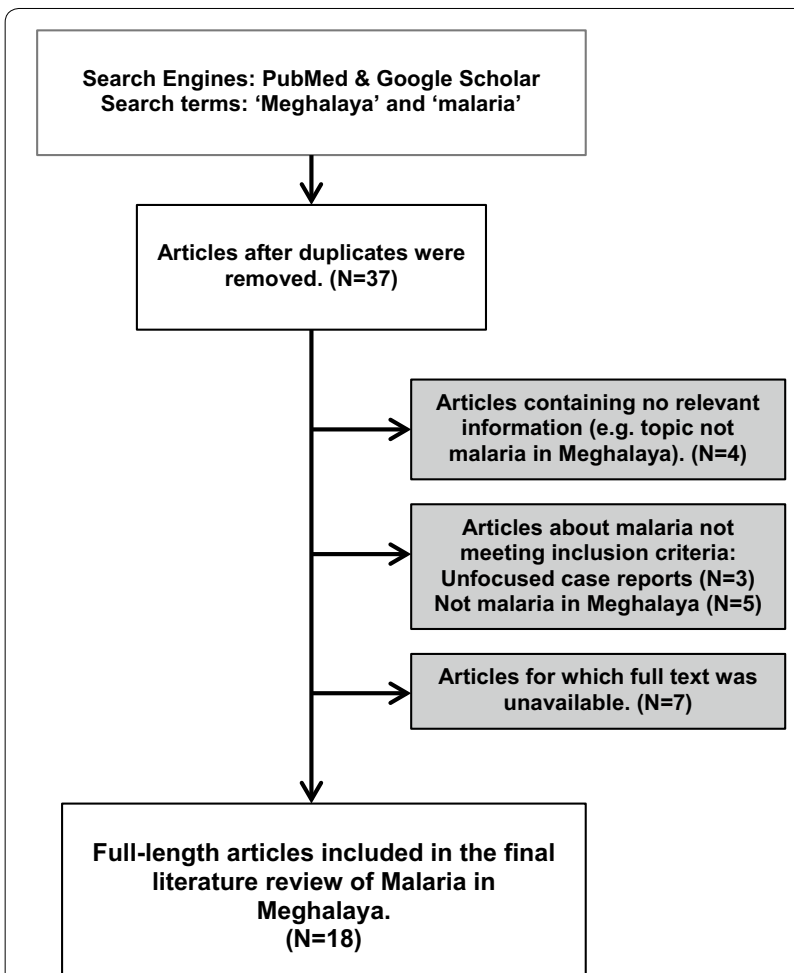

Fig. 2 Selection process of malaria in Meghalaya articles for literature review rainy ('monsoon') season which peaks during the months of May-July [11] but can extend into SeptemberNovember [10]. While both $P$. falciparum and $P$. vivax were found across the state, $P$. falciparum was described as predominant in most regions $[11,13]$. A retrospective analysis of $P$. vivax across all northeastern states from 2008 to 2013 indicated that $P$. vivax comprises 3-8\% of malaria cases in regions of Meghalaya, making it less of a contributor to the malaria burden there than in neighbouring Indian states [13]. The authors also conducted malaria surveys from 1991 to 2012 to identify differences in $P$. vivax infection rates by age group, and found higher rates of $P$. vivax in children $(0-15$ years old $)$ than in adults (15+ years of age) [13]. Mixed-species infections have also been reported in Meghalaya [14].

The northeastern states of India bridge the mainland to regions of east and south-east Asia, providing an avenue for the spread of parasite drug resistance into India. Current treatment recommendations for P. falciparum infections in India are 3 days of artemisinin-based combination therapy (ACT) and a single dose of primaquine on the second day of treatment [15]. However, whereas artesunate and sulfadoxine-pyrimethamine (ASP) are used in mainland India, the current guidelines for the northeastern states-including Meghalaya-recommend artemether-lumefantrine (AL) for $P$. falciparum infections [15]. This change in treatment guidelines occurred in 2013 [16] following growing reports of failed ASP reported in numerous northeastern states, including Mizoram, Tripura, Assam, and Arunachal Pradesh [17, 18]. The literature search revealed documented cases of severe malaria in Meghalaya that were successfully resolved using artesunate [19], but the degree of drug resistance to ACT in the state remains largely undocumented. The recommended treatment for $P$. vivax infection across the whole of India is chloroquine [15], which until 2004 was used to treat $P$. falciparum infections before the emergence of resistance, documented in northeast India [10, 11], Meghalaya, and adjacent states [13].

Although the majority of documented malaria cases in Meghalaya in the last decade are uncomplicated (i.e., cause mild disease), severe clinical presentations involving multiple organs including the central nervous, renal, hepatic, gastrointestinal, and pancreatic systems, have been reported in children [14] and adults [19]. Children (ages $0-17$ years) with severe and unusual malaria syndromes were identified through a retrospective review of children admitted to the pediatric intensive care unit at NEIGHRIMS hospital in Shillong between 2006 and 2009 [14]. Of the 162 malaria cases identified, $10 \%(n=16)$ had unusual, complicated syndromes including seven cases of cerebral malaria and eleven mixed species infections [14]. 
At the same hospital, a more recent report of a 65 year old man presenting with compounding, severe malaria complications was described [19]. While malaria-associated fatalities are relatively infrequent in Meghalaya, they continue to be reported [10,20].

West Garo Hills (WGH) is the western most district in Meghalaya and routinely reports the highest number of malaria cases (nearly $100 \%$ P. falciparum) and malaria deaths. In 2009 and 2010, 72 and 32 deaths respectively were reported in the district through routine monitoring. A survey of $32 \mathrm{WGH}$ villages in 2010 using different modeling approaches and validation of cause of death by verbal autopsy (e.g., confirmation of symptoms experienced before fatal outcome) was performed to determine a more accurate mortality rate and estimate the degree of under-reporting [20]. The models predicted that degree of under-reporting was 7-12 times, and that the true number of malaria-associated deaths was of the same order of magnitude as the World Health Organization estimate that same year ( 2009 WHO estimate $=452$ versus 72 reported) [20]. A retrospective review of malaria rates from 2001 to 2009 conducted in the same district also indicated an overwhelming contribution from $P$. falciparum (98\% of confirmed positives; majority of cases for all months and years in the study period) relative to $P$. vivax, and a high annual parasite index (API, range 12.968.3) throughout the study period [11].

\section{Literature review of malaria vectors in Meghalaya}

Similar to epidemiology studies in Meghalaya, publications concerning malaria vectors in the state were sparse, although some extrapolation can be made from general studies of the northeastern states. For example, in the northeastern states, 23 different Anopheles vectors have been reported, highlighting the complexity of malaria in this region as a whole [10]. In general, Anopheles minimus was described as the major vector of the northeast alongside Anopheles dirus and Anopheles fluviatilis, which are thought to play minor but consistent roles in transmission [10, 11]. A state-wide survey of larvae and adult mosquitos conducted in Meghalaya in the 1980s identified 42 species belonging to six genera of which Anopheles was dominant [21]. Between 2001 and 2009, a review of mosquito prevalence and characterization conducted via daytime indoor collection and nighttime human landing catches in West Garo Hills identified the presence of An. minimus (density per person hour $=3.18$ ) and zoophilic Anopheles aconitus (DPPH $=3.12)$ in high numbers [11]. The authors reported An. minimus as the main vector in the district with a sporozoite positive rate of 2.3\%; all other vectors were sporozoite negative [11]. Anopheles minimus peak biting activity occurred between midnight and 02:00 $\mathrm{h}$ [11].
Meghalaya's Anopheles population is diverse and complex and contains other potential malaria vector species. A survey conducted in northeastern India that included 25 villages spanning East Khasi Hills and East Garo Hills identified an abundance of Anopheles culicifacies vectors (95.7\% of specimens collected) in addition to An. minimus (2.2\%), An. fluviatilis (1.9\%), and An. dirus (0.2\%) [22]. From the 1564 Meghalaya mosquito specimens collected, 63 were positive for Plasmodium by ELISA, and 61 of the infected mosquitoes were An. culicifacies, incriminating it as a major vector in this region [22]. Another study of 10 villages from two regions along the Assam-Meghalaya border, including the Khasi Hills region of Meghalaya, identified Anopheles annularis and Anopheles philippinensis/nivipes (also reported in a Indian-wide survey that included Meghalaya [23]), to be the predominant vector species, with per trap night densities (PTND) of 4 and 4.1 respectively relative to $A n$. minimus (PTND =1.4) [24]. While An. annularis has historically been viewed as zoophilic, the study by Dhiman et al. found that $21.1 \%$ of blood-fed females fed on human blood and reported an overall anthropophilic index of $17.6-23.8 \%$ between sites [24]. The study found only one An. annularis mosquito infected with P. falciparum (2.6\% infection rate) and did not claim a role for the species in malaria transmission.

A study of An. minimus and An. dirus in July-October of 2004 collected using CDC light traps in Williamnagar, East Garo Hills, used PCR of the ITS2 marker gene to identify species within these species complexes. An. minimus species A and An. dirus species D, now formally named An. minimus and Anopheles baimaii, respectively, were identified in Meghalaya and in adjacent northeastern states as well [25]. Further evaluation of the An. dirus complex in the northeast region confirmed a dominant presence of $A n$. baimaii at forest fringes in all seven states, although the authors reported a relatively lower abundance of this sub-species in Meghalaya (e.g. smaller proportion of the state's total vector population) compared to neighboring states [26]. Additionally, a comprehensive review of $>8000$ malaria vector specimens collected from seven different countries highlighted the presence and contribution of An. baimaii specifically in Meghalaya [27].

Of importance to understanding vector dynamics are forces such as changes in the environment and ecology and the emergence of insecticide resistance. With respect to the former, a study of Meghalaya-specific data comparing changes in rice cultivation with observed changes in API at the same location between 1972 and 1983 demonstrated a significant dependence of API on the magnitude of rice cultivation, a finding that likely has minimal effect on malaria transmission in regions of the state where the 
stream-breeding species $A n$. minimus is the sole or dominant vector [28]. The study by Akhtar et al. also explored changes in environment and vector prevalence, highlighting changes in forest coverage and the emergence of a previously under-represented vector species (e.g., $A n$. culicifacies) in the northeastern states. Regarding insecticide resistance in the northeastern states and Meghalaya specifically, An. minimus demonstrated susceptibility to both dichlorodiphenyltrichloroethane (DDT, distributed through for example indoor residual spraying, IRS) and malathion (used to treat bed nets) in studies from the early 2000s $[10,11]$. The study conducted by Dhiman et al. in 2011 along the border with Assam found that $A n$. annularis has demonstrated resistance to DDT and lowlevel resistance to deltamethrin [24].

More recently, attention has also been paid to weather patterns and/or landscape changes that may lead to elevated levels of mosquito breeding, increased malaria transmission, and subsequently a need for enhanced or re-oriented control and prevention strategies. Specifically, a study found that increased El Nino intensity and a high Oceanic Nino Index result in an increase in malaria cases in Meghalaya in a given, concurrent year [29]. In India, El Nino oscillations have a demonstrated association with monsoon and rainfall indicators, and the development of such weather patterns could inform state and local control efforts of the need for enhanced distribution and utilization of available prevention tools given the demonstrated association with increased malaria case incidence. In addition to changing weather and climate patterns, alterations in landscape and land use can yield mosquito breeding habitats in cities and regions previously unaffected by malaria (e.g., high altitudes). This is an important consideration for a state like Meghalaya that is hilly with many villages and towns residing at high altitudes. A study conducted between 2008 and 2011 assessed the association between land use and land cover (LULC), and vector density and diversity, to determine the impact of deforestation and urbanization on mosquito breeding habitats statewide [30]. The study found that Anopheles development, diversity, and density was higher in the 16 villages adjacent to 'disturbed land', including urban, highway, and cultivated areas, relative to the four villages in natural, undisturbed areas [30]. The study findings highlight that LULC i.e., urbanization and deforestation, require monitoring to ensure proper malaria control and prevention strategies are in place, specifically in regions that were previously unaffected.

\section{Literature review of malaria prevention and control methods in Meghalaya}

There are different malaria prevention and control efforts underway in Meghalaya that stem primarily from government sources. Central to control efforts is IRS of insecticide, which in Meghalaya is DDT, and the distribution of insecticide-treated bed nets (ITNs). IRS in Meghalaya is performed two times per year with priority for primary health centres (PHCs) and villages with an API $>2[10,11]$. According to the PHC and village level accredited social health activist (ASHA) records, IRS is performed both indoors and outdoors (e.g. on the external siding of homes and shops) to varying degrees, and although statewide data are not available, the acceptance rate of DDT is recognized to be low [31]. During disease outbreaks when there is a rise in malaria cases, focal spraying has also been reported [11]. ITNs (bed nets impregnated with pyrethroids such as deltamethrin), are distributed under the supervision of the state malaria control programme at the PHC level as directed by the central government [10]. More than 900,000 LLINs were distributed on a gradual, rolling basis statewide in Meghalaya for the first time in 2016, where PHCs harbouring villages with the highest API from the previous year(s) received priority. At the PHC level, LLINs were uniformly distributed to all households over a span of days and weeks to maximize per-person coverage (e.g., distributed based on the number and ages of individuals in each household). LLINs, when properly maintained, are effective for three calendar years. A second distribution of LLINs in Meghalaya is scheduled to occur in 2019, dependent on the supply from the central government and distribution through the state health infrastructure. To supplement the IRS and ITNs, health education and self-protection awareness programmes organized by government and non-government organizations are often conducted during the malaria season or during unexpected periods of high transmission intensity [11].

In addition to the government-distributed control measures, personal mosquito repellents-including coils, vaporizers, mild repellent creams, and mats-represent a $\$ 1.5$ billion industry in India [32] and are available across the state of Meghalaya. Little research has been done to confirm the utilization and effectiveness of repellent products nationally, but one multi-site study in mainland India found that utilization varies widely by household and individual and is associated with higher socio-economic status and level of education [32]. With regards to effectiveness, the study found that the use of mosquito repellents was associated with absence of malaria in some of the participant groups, but overall no consistent observation or association was observed [32]. In Meghalaya, no studies have been conducted regarding the efficacy of personal protection methods. A survey of 200 households conducted in two neighborhoods in Shillong, the capital of Meghalaya, in 2013 indicated that 42\% of households surveyed use mosquito coils in comparison 
to $3.9 \%$ that reported burning tactics (e.g. burning of leaves, wood, or cloth to generate smoke), 13\% that indicated bed net use, and $41.1 \%$ that adopted no measures [31]. While informative, the study was conducted in an urban setting in East Khasi Hills, the district with the least malaria prevalence, and likely sampled a population of people living at a higher SES and with a higher education level than what would be expected statewide, specifically in the villages most affected by malaria.

Timely and effective treatment for clinical malaria is imperative to disease transmission and thus also key for malaria prevention and control. The survey by Battacharyya et al. [31] also evaluated treatment-seeking behaviour, revealing that $97 \%$ of those surveyed indicated that they sought treatment at a regulated facility $(66.9 \%$ government hospitals and $30.1 \%$ private hospital/doctor) with only $3 \%$ reporting self-medication. Primary, facilitybased treatment in Meghalaya involves both active detection (i.e., malaria clinics at the $\mathrm{PHC}$ or village level) and passive detection (i.e., symptomatic individuals come to the PHC) [10]. In addition to facility-administered treatment, alternative medicinal approaches have been reported across the northeastern states. Specifically, interviews with traditional healers and village elders indicated that people use $>65$ different plants belonging to more than 38 plant families to treat malaria [33]. In Meghalaya, the use of roots, leaves, bark, whole plants (oral), root powder (oral), leaf oil (oral), and leaves (chewed with betel nut leaves) was reported for malaria treatment [33]. Crude preparations are typically prepared by boiling/decoction of plants or plant parts in water to ease ingestion [33]. Individuals who self-medicate use similar alternative approaches as opposed to the recommended, hospital administered drug treatment(s), most likely due to availability and cost.

\section{Analysis of Meghalaya State Malaria Control Programme 2015-2017 and the impact of LLINs}

At the state level, malaria cases and malaria-related deaths in Meghalaya have decreased over the last 11 years (Fig. 3). Changes in malaria policy, including the change from chloroquine treatment to antimalarial combination therapy and/or the statewide distribution of LLINs in 2016, indicated in Fig. 3, are most likely responsible for this observation. While continued surveillance and reporting is necessary to determine the true impact of the LLINs, the decline in malaria case incidence and fatalities during the distribution time frame is visually clear (Fig. 3). To assess the suspected impact of mass LLIN distribution in the state and improve understanding of the current malaria picture in Meghalaya, total malaria cases, species-specific malaria cases ( $P$. falciparum and $P$. vivax), and malaria deaths were quantified and compared by district and year for 2015 (year preceding LLIN

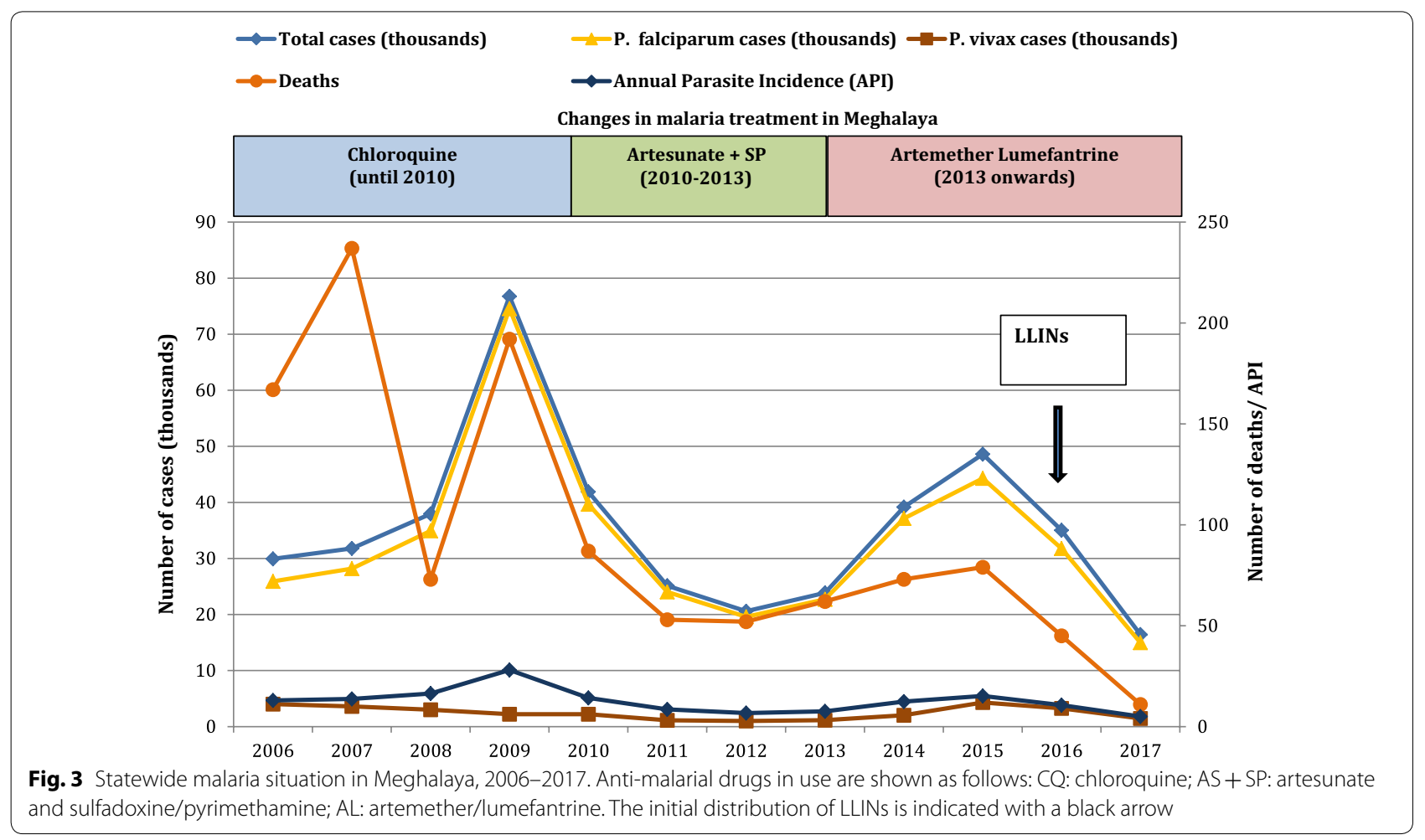


distribution), 2016 (year of LLIN distribution), and 2017 (year following LLIN distribution) in the seven malarious Meghalaya districts (note that East and West Jaintia Hills were combined for analysis). Summary data was first plotted to depict changes and trends over the time frame of interest. P. falciparum was found to be the dominant cause of malaria in four districts $(\approx 90 \%$ or more of infections present in West Garo Hills, East Garo Hills, South Garo Hills, and West Khasi Hills), whereas P. vivax was more common in Jaintia Hills $(\approx 50 \%)$ and Ri Bhoi $(\approx 40 \%)$ (Fig. 4, panels 1-6). Although malaria cases and deaths were still reported in 2017, a steady decline in both malaria cases and deaths was observed in both West and East Garo Hills across the three-year time frame (Fig. 4, panels 1-2). In contrast, South Garo Hills and
West Khasi Hills maintained comparable levels of both malaria cases and deaths from 2015 to 2017 and 20152016, respectively (Fig. 4, panels 3-4). In Ri Bhoi and Jaintia Hills, the two districts where $P$. vivax is present, malaria cases and deaths declined incrementally each year from 2015 to 2017, with cases and deaths dropping severely to single digit numbers in Ri Bhoi by 2017 (Fig. 4, panels 5-6). As outlined in the literature review results, malaria in Meghalaya is persistent and perennial but rises during the monsoon season and summer months of June to September. Despite the decline in overall and speciesspecific malaria cases, this cyclic trend was observed for total malaria cases, $P$. falciparum cases, and $P$. vivax cases (when present) across all 3 years (Fig. 4).
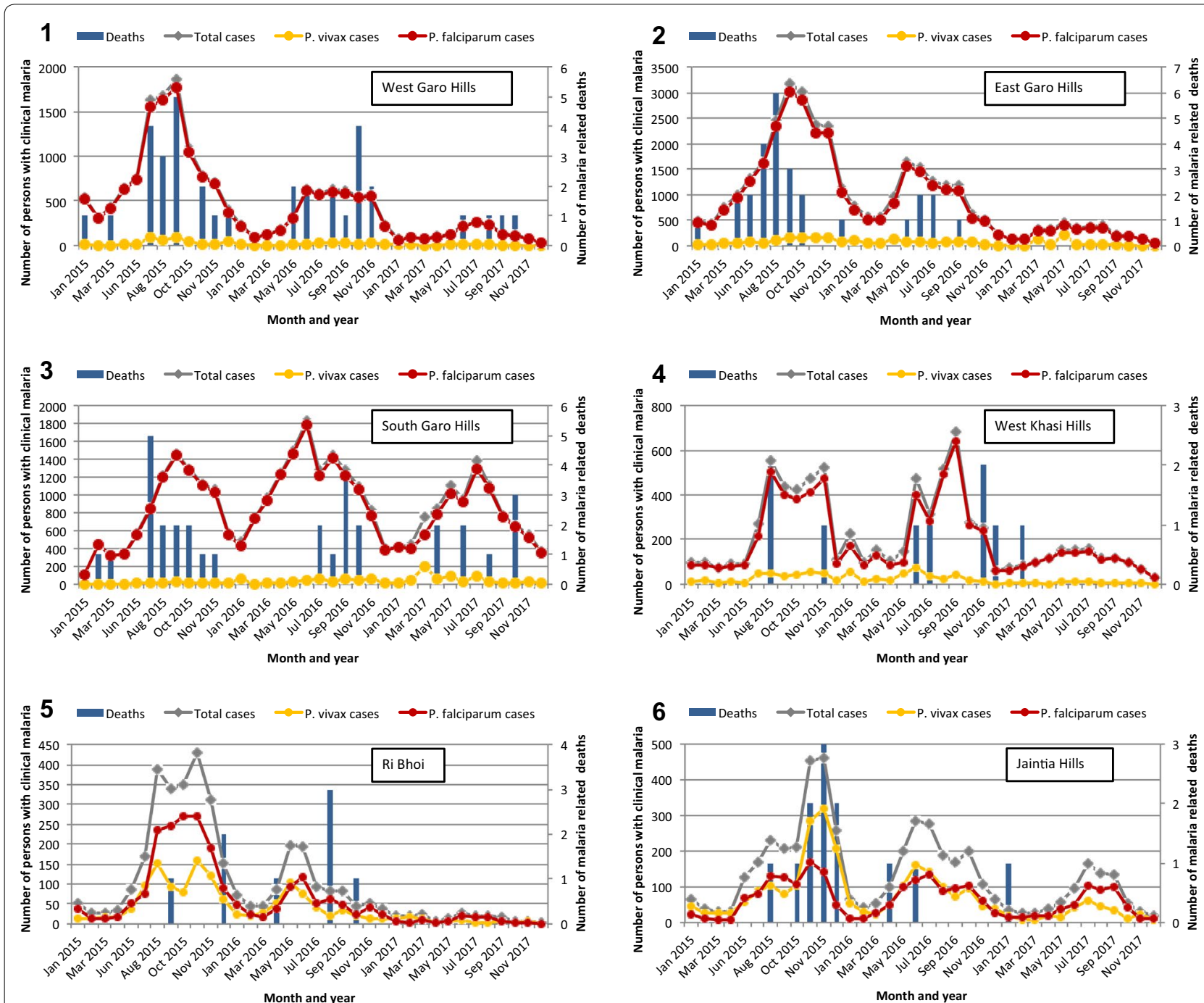

Fig. 4 Monthly malaria cases in six districts in Meghalaya, 2015-2017. (1) West Garo Hills, (2) East Garo Hills, (3) South Garo Hills, (4) West Khasi Hills, (5) Ri Bhoi, (6) Jaintia Hills 
Key to understanding malaria epidemiology and changes in case incidence in a given region are ageand sex-specific infection distributions following a new intervention. In all six districts, the majority of malaria infections were diagnosed among persons $\geq 15$ years (range $42-64 \%$ ), with young children (0-4 years) contributing $12-25 \%$ of all infections, and older children (5-14 years) contributing $21-32 \%$ (Table 1). By gender and age in 2015, males dominated in the age group $\geq 15$ years in West Garo Hills $(55.7 \%$ males compared to $50.9 \%$ males in age group $<15$ years, $\mathrm{p}<0.001)$, West Khasi Hills $(54.7 \%$ versus $49.8 \%, \mathrm{p}=0.006)$, Ri Bhoi $(58.1 \%$ versus $52.8 \%$, $\mathrm{p}=0.009)$ and Jaintia Hills $(59.1 \%$ versus $51.2 \%$, $\mathrm{p}<0.001$ ), whereas females in the age group $\geq 15$ years were more likely to have malaria in East (51.4\% female among $\geq 15$ years compared to $49.5 \%$ female in age group $<15$ years, $\mathrm{p}=0.007)$ and South Garo Hills (54.4\% female among $\geq 15$ years compared to $49.7 \%$ female in age group $<15$ years, $\mathrm{p}<0.001)$, the districts with the highest API (Table 1).

A dramatic reduction in malaria cases in West and East Garo Hills, Ri Bhoi, and Jaintia Hills occurred in 2016, the same year that the first distribution of LLINs occurred (Table 1: reductions in reported malaria of $56.2 \%, 45.2 \%, 56.4 \%$, and $23.8 \%$ respectively relative to 2015), and continuing in 2017 (again relative to 2015 , reductions in reported malaria of $85.6 \%$, $84.7 \%, 92.1 \%$, and $64.5 \%$ respectively) as evident by the plotted data (Fig. 4). These reductions were also reflected in the API (Fig. 5). In West Khasi Hills, the decline in reported malaria occurred mainly in 2017 (e.g., reduction of $60.8 \%$ relative to 2015 ; Fig. 4). No reduction was noted in South Garo Hills in the years examined. Compared to 2015, the 2017 reported proportion of persons $\geq 15$ years of age with malaria significantly increased in all districts except for South Garo Hills (Fig. 5a and Table 1; West Garo Hills from $56.1 \%$ in 2015 to $64 \%$ in 2017, p $<0.001$; East Garo Hills 42.1-47.2\%, $\mathrm{p}<0.001$; South Garo Hills $47.1 \%$ in 2015 and 2017, $\mathrm{p}=0.670$, West Khasi Hills 54.4-64.1\%, $\mathrm{p}<0.001$; Ri Bhoi 48.7-59.1\%, $\mathrm{p}=0.006$; and Jaintia Hills 51-64.2\%, $\mathrm{p}<0.001$ ). Gender differences among age groups were not significant in West Khasi Hills, Ri Bhoi, and Jaintia Hills, but persisted in 2017 in the other districts in the same direction as 2015 (Fig. 5b, e.g. West Garo Hills 56.7\% males in age group $\geq 15$ years compared to $50.4 \%$ males in age group $<15$ years, $\mathrm{p}=0.017$, East Garo Hills $55.5 \%$ female among $\geq 15$ years compared to $49.5 \%$ females in age group $<15$ years, $\mathrm{p}=0.001$ and South Garo Hills $57.4 \%$ female among $\geq 15$ years and $50.9 \%$ in age group $<15$ years, $\mathrm{p}<0.001)$.

\section{Discussion/conclusions}

This is the first systematic review of malaria epidemiology and transmission in the northeastern state of Meghalaya. In line with the Indian mainland [34], a review of the available literature on malaria in Meghalaya confirms an overall theme: it is a complex disease which has many different factors combining to ensure continued transmission, making control and prevention challenging. While $P$. falciparum malaria certainly dominates, both $P$. vivax and mixed infections are common, and severe, unusual, and fatal cases have all been reported in recent years $[11,13,14,19,20]$. The overwhelming diversity of key, emerging, and potential mosquito vectors, including $A n$. minimus, $A n$. baimaii, An. culicifacies, An. annularis, and An. fluviatilis amongst several others [21-25], only adds to the complexity of malaria transmission and epidemiology across the state. These reports highlight the need for continued surveillance and increased focus on both prevention methods and early diagnosis.

A second theme from the malaria in Meghalaya literature was that most studies were focused on a single district or region $[11,20]$ making them difficult to generalize to the state at large. For example, the study by Sharma et al. [13] reported a low $P$. vivax burden in the state of Meghalaya in comparison to other northeastern states. However, the study sites were located in the West Garo Hills district, which is known for dominant $P$. falciparum malaria and also geographically distant from the districts in Meghalaya known to harbor higher numbers of P. vivax malaria cases (e.g., Jaintia Hills, Ri Bhoi). Many of the vector reports from the state were also targeted to specific regions $[11,22,26]$. Taken together, the literature covering malaria epidemiology and transmission in Meghalaya is piecemeal at best. While it is clear that malaria in Meghalaya is complex, the differences in Plasmodium species prevalence and distribution, land use and ecology, and vector species across the state make it challenging to generalize trends from the existing epidemiology and vector literature alone.

Malaria prevention and control methods in Meghalaya are also diverse in nature and seemingly widespread across the state. Distributed interventions such as IRS, impregnated bed nets, and education/awareness programmes are commonly employed, although poor uptake of certain interventions (e.g., IRS) was reported [31]. However, the malaria prevention and control literature lacks studies that determine and characterize the effectiveness of interventions and control methods in use in Meghalaya. Such studies will become more important as malaria numbers decrease, requiring focused, contextspecific efforts (e.g., village by village, region by region) to obtain gains until elimination is achieved. 


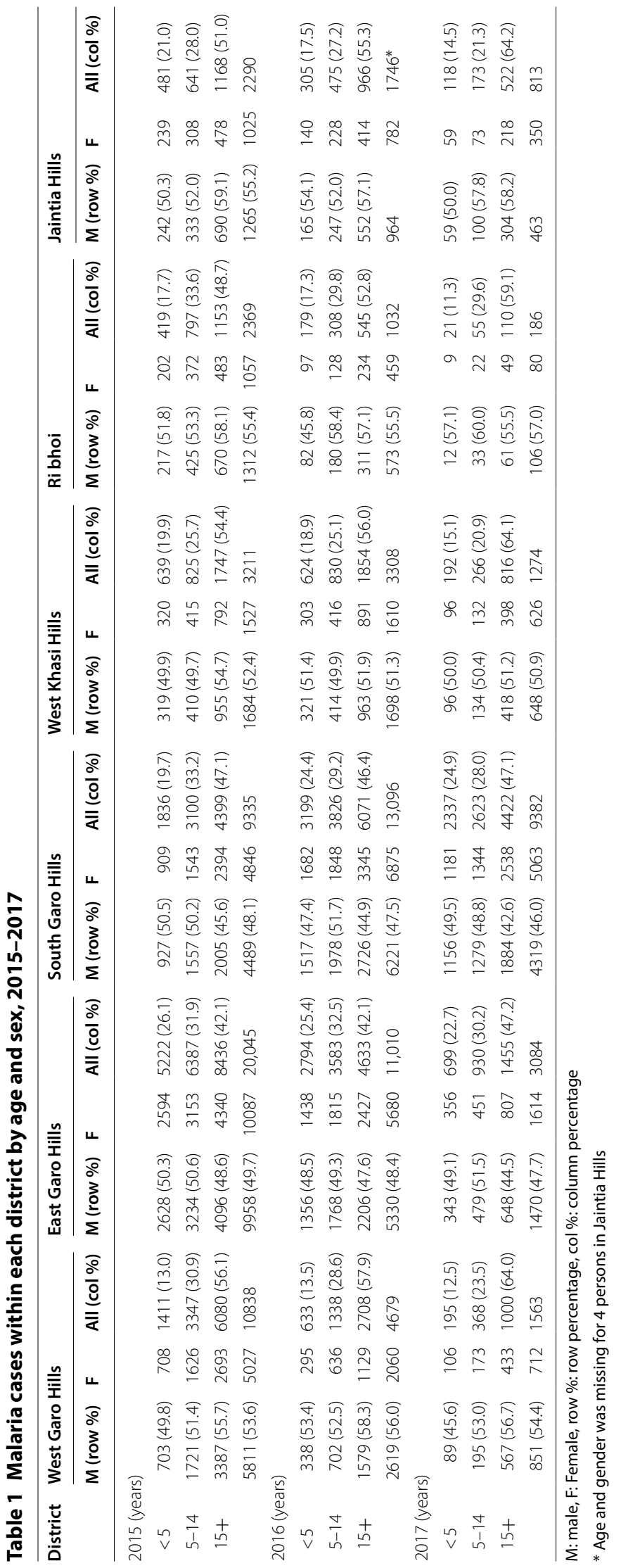



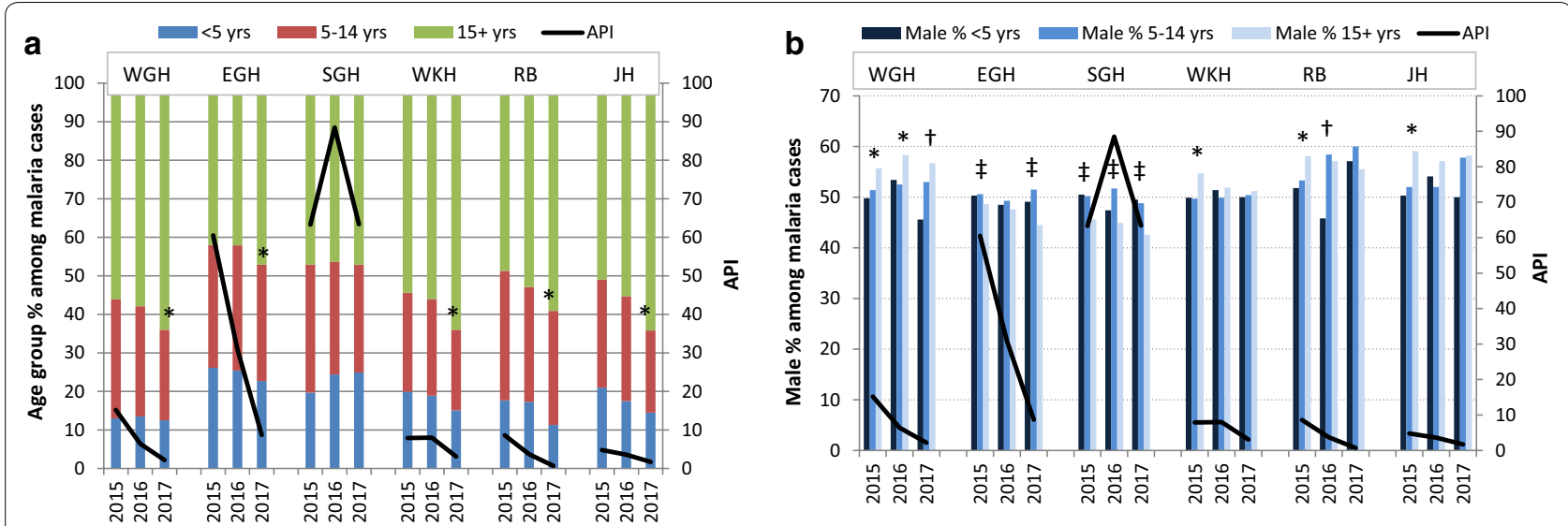

Fig. 5 Clinical malaria by age and sex, 2015-2017. a Percentage of malaria cases (y-axis) for each age group (<5 years, 5-14 years, 15+ years) in each district; WGH: West Garo Hills, EGH: East Garo Hills, SGH: South Garo Hills, WKH: West Khasi Hills, RB: Ri Bhoi, JH: Jaintia Hills; *Denotes significance $(p<0.05)$ in a comparison of age group 15+ versus children (<5 years, 5-14 years) in 2015 versus 2017 . b The proportion of males among each age group for each year is shown for each district. *Denotes proportion of males in $15+$ years group is significantly higher compared to males in 5-14 years age group or males in < 5 years age group; ${ }^{\dagger}$ Denotes proportion of males in $15+$ years group is significantly higher compared to males in < 5 years age group but not to males in 5-14 years age group; ${ }^{\ddagger}$ Denotes proportion of males in $15+$ years group is significantly less compared to males in 5-14 years age group and to males in $<5$ age years age group; The Z-axis represents the API in both panels

While $A n$. minimus and An. baimaii are likely to continue playing the major role in malaria transmission in Meghalaya in the forseeable future, landcover change appears to be increasing the complexity of malaria epidemiology in this region. To achieve malaria elimination, characterization of all relevant vectors and their varying ecologies and behaviours is essential if interventions are to be correctly targetted and ultimately effective. Furthermore, heightened awareness of the potential for mosquito adaptation to malaria interventions (e.g., switch to earlier biting times before bed net use as recorded for several Anopheles species [35]; greater exophily [36]), specifically the rollout of LLINs [37] in Meghalaya, is also key to gaining and maintaining vector control in this region. Overall, a fuller understanding of vector complexity and malaria transmission dynamics is essential for the integrated vector management approach that will likely be required for eventual malaria elimination [38].

Analysis and visual presentation of the Meghalaya State Malaria Control Programme data from 2015 to 2017 highlights the reductions in malaria cases and fatalities in five of the six districts analysed since the distribution of $>900,000$ LLINs across the state. While these data are highly promising, it is not possible to determine if the introduction of LLINs directly caused the reduction in malaria cases or in combination with other control measures or factors, and further studies are required to evaluate their efficacy. Asymptomatic malaria rates and distribution, changes in vector and parasite species prevalence and distributions, and the emergence or expansion of drug resistant vector populations are all important and relevant factors requiring study. Interestingly, a significant reduction in proportion of infected children relative to adults in 2017 (post-LLIN distribution) relative to 2015 (pre-LLIN distribution) across 5 of the 6 districts analysed might suggest that children were given priority within a family dwelling for LLIN coverage. The one exception to the reduction in malaria cases seen during 2016-2017 is South Garo Hills, which had consistently high numbers of cases during the 2016 and 2017 monsoon seasons. Many of the villages in South Garo Hills are difficult to reach by car, and regions along the Meghalaya-Bangladesh border are areas that experience civil unrest. In combination, these features make the distribution of interventions such as LLINs and education/awareness programmes challenging, and may have resulted in poor distribution or use of LLINs in 2016.

Overall, the paucity of comprehensive articles on malaria in Meghalaya makes it difficult to develop a complete picture of the epidemiology, transmission and control of the disease within the state. Indeed, the regional differences in API, Plasmodium species prevalence and distribution, population demographics (sex, gender), and malaria-associated fatality rates from 3 years of surveillance data highlights the heterogeneous nature of malaria in Meghalaya, and underscores how generalizing information from one district or region of Meghalaya to another is not recommended. District-wise surveillance of malaria in Meghalaya as conducted by the Meghalaya State Malaria Control Programme is essential to understanding malaria epidemiology statewide and for designing and conducting 
studies to improve surveillance, intervention efficacy, and disease management. While it is important to consider the limitations of surveillance data and state level reporting (e.g. under/over reporting; missing districts), continued monitoring-including both active and passive surveillance-and routine follow up at the local level is required to ensure the accuracy of reported data and consistency across districts in order to maximize the impact of downstream prevention and control efforts.

\begin{abstract}
Abbreviations
ACT: artemisinin-based combination therapy; API: annual parasite index; ASHA: accredited social health activist; DDT: dichlorodiphenyltrichloroethane; DPPH: density per person hour; ITNs: insecticide-treated nets; IRS: indoor residual spray; LLINs: long-lasting insecticide-treated bed nets; LULC: land use and land cover; NVBDCP: National Vector Borne Disease Control Programme; PHC: primary health centre; PTND: per trap night density; SES: socioeconomic status.
\end{abstract}

\section{Authors' contributions}

$A K, J C$, and SA wrote the manuscript with input from all authors and vector text contribution from $C W ; L J$ and AVE undertook data collection and analysis; AVE performed literature searches; AK and AVE read, summarized, and categorized all literature from the defined search; AK and AVE generated the figures and tables. All authors read and approved the final manuscript.

\section{Author details}

${ }^{1}$ Center for Genomics and Systems Biology, Department of Biology, New York University, New York, NY, USA. ${ }^{2}$ Indian Institute of Public Health-Shillong, Lawmali, Shillong, Meghalaya, India. ${ }^{3}$ University of Manchester, Manchester, UK. ${ }^{4}$ Martin Luther Christian University, Shillong, Meghalaya, India.

\section{Acknowledgements}

We thank the Department of Health, Government of Meghalaya and the Meghalaya State Programme Management Unit, NVBDCP for access to malaria surveillance data. Specifically, we would like to acknowledge Additional Chief Secretary Shri PW Ingty IAS, Health Secretary Shri Pravin Bakshi IAS, the Deputy Director DHS and SPO Dr. R Lyngdoh, and M\&E consultant Larry Kharbamon for facilitating data sharing and providing the API map of Meghalaya. We also thank the Center for the Study of Complex Malaria in India (CSCMI) personnel based at the Indian Institute of Public Health-Shillong for organizing and collating the data.

\section{Competing interests}

The authors declare that they have no competing interests.

\section{Availability of data and materials}

Data generated or analysed in this study are included within the article. Malaria surveillance data was sourced from the Meghalaya State Malaria Control Programme.

\section{Consent for publication}

Not applicable.

\section{Ethics approval and consent to participate} Not applicable.

\section{Funding}

Research reported in this publication was supported by the National Institute of Allergy and Infectious Diseases of the National Institutes of Health under Award Number U19A1089676 as part of the International Centers for Excellence in Malaria Research. The content is solely the responsibility of the authors and does not necessarily represent the official views of the National Institutes of Health.

\section{Publisher's Note}

Springer Nature remains neutral with regard to jurisdictional claims in published maps and institutional affiliations.

Received: 6 September 2018 Accepted: 1 November 2018

Published online: 06 November 2018

\section{References}

1. Rajagopal R. Studies on persistent transmission of malaria in Burnihat, Meghalaya. J Commun Dis. 1976;8:235-45.

2. Karlsson BG. Anthropology and the "Indigenous Slot": claims to and debates about Indigenous Peoples'Status in India. In: Karlsson BG, Tanka Bahadur S, editors. Indigeneity in India. London: Kegan Paul Publ; 2006. p. $51-73$.

3. India Government. National commission for scheduled tribes. New Delhi: Booklet; 2018.

4. Sharma RK, Thakor HG, Saha KB, Sonal GS, Dhariwal AC, Singh N. Malaria situation in India with special reference to tribal areas. Indian J Med Res. 2015;141:537-45.

5. Ministry of Tribal Affairs. Demographic status of scheduled tribe population of India. 2013. Government of India. http://www.tribal.gov.in. Accessed 11 Oct 2013.

6. Myers N, Mittermeier RA, Mittermeier CG, da Fonseca GA, Kent J. Biodiversity hotspots for conservation priorities. Nature. 2000;403:853-8.

7. Albert S. Indigenous peoples, food, and the environment in northeast India. The Routledge Handbook of Food Ethics. Rawlinson: Routledge; 2017. p. 113-24.

8. Lele N, Joshi PK. Analyzing deforestation rates, spatial forest cover changes and identifying critical areas of forest cover changes in NorthEast India during 1972-1999. Environ Monit Assess. 2009;56:159-70.

9. Albert S, Nongrum M, Webb EL, Porter JD, Kharkongor GC. Medical pluralism among indigenous peoples in northeast India-implications for health policy. Trop Med Int Health. 2015;20:952-60.

10. Dev V, Bhattacharyya PC, Talukdar R. Transmission of malaria and its control in the northeastern region of India. J Assoc Physicians India. 2003;51:1073-6.

11. Dev V, Sangma BM, Dash AP. Persistent transmission of malaria in Garo hills of Meghalaya bordering Bangladesh, north-east India. Malar J. 2010:9:263.

12. Dev V. Anopheles minimus: its bionomics and role in the transmission of malaria in Assam, India. Bull World Health Organ. 1996;74:61-6.

13. Sharma VP, Dev V, Phookan S. Neglected Plasmodium vivax malaria in northeastern states of India. Indian J Med Res. 2015;141:546-55.

14. Dass R, Barman H, Duwarah SG, Deka NM, Jain P, Choudhury V. Unusual presentations of malaria in children: an experience from a tertiary care center in North East India. Indian J Pediatr. 2010;77:655-60.

15. National Institute of Malaria Research. Guidelines for diagnosis and treatment of malaria in India. New Delhi: Government of India; 2014.

16. Anvikar AR, Arora U, Sonal GS, Mishra N, Shahi B, Savargaonkar D, et al. Antimalarial drug policy in India: past, present \& future. Indian J Med Res. 2014;139:205-15.

17. Mishra N, Kaitholia K, Srivastava B, Shah NK, Narayan JP, Dev V, et al. Declining efficacy of artesunate plus sulphadoxine-pyrimethamine in northeastern India. Malar J. 2014;13:284.

18. Mishra N, Srivastava B, Bharti RS, Rana R, Kaitholia K, Anvikar AR, et al. Monitoring the efficacy of antimalarial medicines in India via sentinel sites: outcomes and risk factors for treatment failure. J Vector Borne Dis. 2016;53:168-78.

19. Barman B, Bhattacharya PK, Lynrah KG, Ete T, Issar NK. Acute pancreatitis in a patient with complicated falciparum malaria. J Clin Diagn Res. 2016;10:18-20.

20. Bhattacharjee J, Sangma BM, Dhariwal AC, Bandyopadhyay S. Estimation of malaria mortality of a district in India during 2010 by using three models. J Commun Dis. 2012;44:1-7.

21. Das SC, Das NG, Baruah I. Mosquito survey in Meghalaya. Indian J Public Health. 1984;28:147-51. 
22. Akhtar N, Nagpal BN, Kapoor N, Srivastava A, Valecha N. Role of An. culicifacies as a vector of malaria in changing ecological scenario of Northeastern states of India. J Vector Borne Dis. 2016;53:264-71.

23. Subbarao SK, Kumar KV, Nanda N, Nagpal BN, Dev V, Sharma VP. Cytotaxonomic evidence for the presence of Anopheles nivipes in India. J Am Mosq Control Assoc. 2000;16:71-4.

24. Dhiman S, Rabha B, Goswami D, Das NG, Baruah I, Bhola RK, et al. Insecticide resistance and human blood meal preference of Anopheles annularis in Asom-Meghalaya border area, northeast India. J Vector Borne Dis. 2014;51:133-6.

25. Prakash A, Walton C, Bhattacharyya DR, Loughlin SO, Mohapatra PK, Mahanta J. Molecular characterization and species identification of the Anopheles dirus and An. minimus complexes in north-east India using r-DNA ITS-2. Acta Trop. 2006;100:156-61.

26. Prakash A, Sarma DK, Bhattacharyya DR, Mohapatra PK, Bhattacharjee K, Das K, et al. Spatial distribution and r-DNA second internal transcribed spacer characterization of Anopheles dirus (Diptera: Culicidae) complex species in north-east India. Acta Trop. 2010;114:49-54.

27. Sallum MA, Peyton EL, Wilkerson RC. Six new species of the Anopheles leucosphyrus group, reinterpretation of An. elegans and vector implications. Med Vet Entomol. 2005;19:158-99.

28. Sharma VP, Srivastava A, Nagpal BN. A study of the relationship of rice cultivation and annual parasite incidence of malaria in India. Soc Sci Med. 1994;38:165-78.

29. Dhiman RC, Sarkar S. El Nino Southern Oscillation as an early warning tool for malaria outbreaks in India. Malar J. 2017;16:122.

30. Srivastava AK, Kharbuli B, Shira DS, Sood A. Effect of land use and land cover modification on distribution of anopheline larval habitats in Meghalaya. India. J Vector Borne Dis. 2013;50:121-6.
31. Bhattacharyya $\mathrm{H}$. Knowledge, beliefs, and practices regarding malaria in urban setting of East Khasi Hills district, Meghalaya. Int J Med Sci Public Health. 2015:4:1042-5.

32. van Eijk AM, Ramanathapuram L, Sutton PL, Peddy N, Choubey S, Mohanty S, et al. The use of mosquito repellents at three sites in India with declining malaria transmission: surveys in the community and clinic. Parasit Vectors. 2016:9:418.

33. Bora U, Sahu A, Saikia AP, Ryakala VK, Goswami P. Medicinal plants used by the people of Northeast India for curing malaria. Phytother Res. 2007;21:800-4.

34. Das A, Anvikar AR, Cator LJ, Dhiman RC, Eapen A, Mishra N, et al. Malaria in India: the center for the study of complex malaria in India. Acta Trop. 2012;121:267-73

35. Sougoufara S, Diedhiou SM, Doucoure S, Diagne N, Sembene PM, Harry $M$, et al. Biting by Anopheles funestus in broad daylight after use of longlasting insecticidal nets: a new challenge to malaria elimination. Malar J. 2014;13:125.

36. Killeen GF. Characterizing, controlling and eliminating residual malaria transmission. Malar J. 2014;13:330.

37. Huijben S, Paaijmans KP. Putting evolution in elimination: winning our ongoing battle with evolving malaria mosquitoes and parasites. Evol Appl. 2018;11:415-30.

38. Benelli G, Beier JC. Current vector control challenges in the fight against malaria. Acta Trop. 2017;174:91-6.
Ready to submit your research? Choose BMC and benefit from:

- fast, convenient online submission

- thorough peer review by experienced researchers in your field

- rapid publication on acceptance

- support for research data, including large and complex data types

- gold Open Access which fosters wider collaboration and increased citations

- maximum visibility for your research: over $100 \mathrm{M}$ website views per year

At BMC, research is always in progress.

Learn more biomedcentral.com/submissions 